\title{
Flow polynomials of a signed graph
}

\author{
Xiangyu Ren Jianguo Qian* \\ School of Mathematical Sciences \\ Xiamen University \\ Xiamen, Fujian 361005, P.R. China \\ 251356374@qq.com, jgqian@xmu.edu.cn
}

Submitted: Oct 5, 2018; Accepted: Aug 9, 2019; Published: Aug 30, 2019

(C) The authors. Released under the CC BY-ND license (International 4.0).

\begin{abstract}
For a signed graph $G$ and non-negative integer $d$, it was shown by DeVos et al. that there exists a polynomial $F_{d}(G, x)$ such that the number of the nowhere-zero $\Gamma$-flows in $G$ equals $F_{d}(G, x)$ evaluated at $k$ for every Abelian group $\Gamma$ of order $k$ with $\epsilon(\Gamma)=d$, where $\epsilon(\Gamma)$ is the largest integer $d$ for which $\Gamma$ has a subgroup isomorphic to $\mathbb{Z}_{2}^{d}$. We define a class of particular directed circuits in $G$, namely the fundamental directed circuits, and show that all $\Gamma$-flows (not necessarily nowhere-zero) in $G$ can be generated by these circuits. It turns out that all $\Gamma$-flows in $G$ can be evenly partitioned into $2^{\epsilon(\Gamma)}$ classes specified by the elements of order 2 in $\Gamma$, each class of which consists of the same number of flows depending only on the order of $\Gamma$. Using an extension of Whitney's broken circuit theorem of Dohmen and Trinks, we give a combinatorial interpretation of the coefficients in $F_{d}(G, x)$ for $d=0$ in terms of broken bonds. Finally, we show that the sets of edges in a signed graph that contain no broken bond form a homogeneous simplicial complex.
\end{abstract}

Mathematics Subject Classifications: 05C21, 05C22, 05C31

\section{Introduction}

Nowhere-zero $\mathbb{Z}_{k}$-flows, or modular $k$-flows, in a graph were initially introduced by Tutte [17] as a dual problem to vertex-colouring of plane graphs. It has long been known that the number of nowhere-zero $\mathbb{Z}_{k}$-flows, or, more generally, nowhere-zero $\Gamma$-flows (flows with values in $\Gamma$ ) for an Abelian group $\Gamma$ of order $k$ is a polynomial function in $k$, which does not depend on the algebraic structure of the group [17]. An analog of a $\mathbb{Z}_{k}$-flow is an integer $k$-flow, or $k$-flow for short, in which values on edges are integers strictly less than $k$ in absolute value. It is well known that a graph has a nowhere-zero $k$-flow if and

*Corresponding author, supported by NSFC grant No.11561058 and 11471273. 
only if it has a nowhere-zero $\mathbb{Z}_{k}$-flow [16]. In [14], Kochol showed that the number of nowhere-zero $k$-flows is also a polynomial in $k$, although not the same polynomial as that for nowhere-zero $\mathbb{Z}_{k}$-flows.

The notion of a signed graph was introduced by Harary [11], initially as a model for social networks. In a similar way to flows in plane graphs, or more generally in graphs embedded in an orientable surface, the definition of $\mathbb{Z}_{k}$-flows in signed graphs is naturally considered for the study of graphs embedded in a non-orientable surface, where nowherezero $\mathbb{Z}_{k}$-flows emerge as the dual notion to local tensions $[2,13]$.

In contrast to ordinary graphs, the problem of counting nowhere-zero flows in a signed graph seems more complicated and there are relatively few results to be found in the literature. Applying the theory of counting lattice points in inside-out polytopes to signed graphs, Beck and Zaslavsky [1] showed that the number of nowhere-zero $k$-flows in a signed graph is a quasi-polynomial of period two, that is, given by a pair of polynomials, one for odd values of $k$ and the other for even $k$. In the same paper, Beck and Zaslavsky also showed that there exists a polynomial $f(G, x)$ such that, for every odd integer $k$, the number of nowhere-zero $\Gamma$-flows in a signed graph $G$ equals $f(G, x)$ evaluated at $k$ for every Abelian group $\Gamma$ with $|\Gamma|=k$. This result was recently extended by DeVos, Rollová and Śámal [6] to a general Abelian group: for any non-negative integer $d$, there exists a polynomial $f_{d}(G, x)$ such that the number of nowhere-zero $\Gamma$-flows in $G$ is exactly $f_{d}(G, x)$ evaluated at $n$ for every Abelian group $\Gamma$ with $\epsilon(\Gamma)=d$ and $|\Gamma|=2^{d} n$, where $\epsilon(\Gamma)$ is the largest integer $d$ for which $\Gamma$ has a subgroup isomorphic to $\mathbb{Z}_{2}^{d}$. More recently, Goodall et al. [9] (available from arXiv) gave an explicit expression for $f_{d}(G, x)$ in the form of an edge-subset expansion.

In this paper we focus on the combinatorial structure of $\Gamma$-flows in a signed graph $G$ and the coefficients in the polynomial $f_{d}(G, x)$. For convenience, instead of working on $f_{d}(G, x)$, we will work on the polynomial $F_{d}(G, x)$ defined by $F_{d}(G, x)=f_{d}\left(G, 2^{-d} x\right)$ and call $F_{d}(G, x)$ the $d$-type flow polynomial, or simply the flow polynomial of $G$. It can be seen that $F_{d}(G, x)$ evaluated at $k$ is exactly the number of nowhere-zero $\Gamma$-flows in $G$ for every Abelian group $\Gamma$ with $\epsilon(\Gamma)=d$ and $|\Gamma|=k$.

In the third section we introduce the fundamental directed circuits and the fundamental root circuit (a particular unbalanced circuit) in a signed graph $G$. We show that every $\Gamma$-flow (not necessarily nowhere-zero) in $G$ can be generated by these circuits. More specifically, the values of the flows assigned to the fundamental directed circuits are elements of $\Gamma$, while the value assigned to the fundamental root circuit is an element of order 2 in $\Gamma$. As a consequence, all $\Gamma$-flows in $G$ can be evenly partitioned into $2^{\epsilon(\Gamma)}$ classes specified by the elements of order 2 in $\Gamma$. Moreover, each class consists of the same number of flows, which depends only on the order of the group. This gives an explanation for why the number of the $\Gamma$-flows in a signed graph varies with different $\epsilon(\Gamma)$ and, also gives an answer to a problem posed by Beck and Zaslavsky in [1]. Further, this result also yields an explicit expression of the polynomial $F_{d}(G, x)$ obtained earlier by Goodall et al.

In the fifth section we give a combinatorial interpretation of the coefficients in $F_{d}(G, x)$ for $d=0$. To this end, we apply Whitney's broken circuit theory [18]. In the study of graph coloring, one significance of Whitney's broken circuit theorem is that it gives a 
very nice 'cancellation' to reduce the terms in the chromatic polynomial (represented in the form of inclusion-exclusion) until the remaining terms cannot be cancelled out any further, and also gives a combinatorial interpretation for the coefficients of the polynomial $[3,4]$. Using an extended form of Whitney's theorem given by Dohmen and Trinks [7], we show that $F_{0}(G, x)$ is a polynomial with leading term $x^{m-n}$ and with its coefficients alternating in sign. More specifically, the coefficient of $(-1)^{i} x^{m-n-i}, i=0,1, \cdots, m-n$, is exactly the number of the sets consisting of $i$ edges that contain no broken bond. As an example, we give an analytic expression of $F_{0}(G, x)$ for a class of signed graphs that contain no balanced circuit. Finally, we show that the broken bonds in a signed graph form a homogeneous simplicial complex of top dimension $m-n$. Thus, the coefficients of $F_{0}(G, x)$ are the simplex counts in each dimension of the complex.

\section{Preliminaries}

Graphs in this paper may contain parallel edges or loops. For a graph $G$, we use $V(G)$ and $E(G)$ to denote its vertex set and edge set, respectively. A signed graph is a pair $\left(G, E_{N}\right)$, where $E_{N} \subseteq E(G)$ and the edges in $E_{N}$ are negative while the other ones are positive. In the following, we will use $G$ simply to denote a signed graph if no confusion can occur.

A circuit is a connected 2-regular graph. An unbalanced circuit in a signed graph is a circuit that has an odd number of negative edges. A balanced circuit is a circuit that is not unbalanced. A signed subgraph is unbalanced if it contains an unbalanced circuit; otherwise, it is balanced. In particular, a subgraph without negative edges is balanced. A barbell is the union of two unbalanced circuits $C_{1}, C_{2}$ and a (possibly trivial) path $P$ with end vertices $v_{1} \in V\left(C_{1}\right)$ and $v_{2} \in V\left(C_{2}\right)$, such that $C_{1}-v_{1}$ is disjoint from $P \cup C_{2}$ and $C_{2}-v_{2}$ is disjoint from $P \cup C_{1}$. We call $P$ the barbell path of the barbell. A signed circuit is either a balanced circuit or a barbell.

Given a signed graph $G$, switching at a vertex $v$ is the inversion of the sign of each edge incident with $v$. Two signed graphs are said to be switching-equivalent if one can be obtained from the other by a series of switchings. It is known $[13,15,23]$ and easy to see that equivalent signed graphs have the same sets of unbalanced circuits and the same sets of balanced circuits. This means, in particular, that a balanced signed graph $G$ is switching-equivalent to the underlying unsigned graph of $G$.

Following [2, 21, 22], we introduce the notion of half-edges so as to orient a signed graph $G$ : each edge $e=u v$ of $G$ is viewed as composed of two half-edges, denoted by $(u, e)$ and $(v, e)$. An orientation $\tau(G)$ of a signed graph $G$ is obtained by orienting all its edges. To orient a half edge $(v, e)$ we mean to assign it with a sign $\tau(v, e)$, meaning that $e$ points toward $v$ if $\tau(u, e)=-1$ and points away $v$ if $\tau(u, e)=1$. An edge $e=u v$ is oriented if both $(u, e)$ and $(v, e)$ are oriented subject to $\tau(u, e) \tau(v, e)=-\sigma(e)$, where $\sigma$ is the sign function defined by $\sigma(e)=1$ when $e$ is positive and $\sigma(e)=-1$ otherwise. Thus, the orientation of a positive edge is in the usual way and, in an orientation of a negative edge $e$, both the two half-edges point toward the end vertices of $e$, called extroverted, or both point toward the inside of $e$, called introverted. For an edge $e=u v$, we also write 
$\tau(u, e)$ and $\tau(v, e)$ together as $\tau(e)$ for short. For a vertex $v$ in an orientation of a signed graph, we denote by $E^{+}(v)$ (resp., $E^{-}(v)$ ) the set of the half edges $(v, e)$ with $\tau(v, e)=1$ (resp., $\tau(v, e)=-1$ ).

Let $D=\tau(G)$ be an orientation of $G$ and $\Gamma$ be an additive Abelian group. A map f : $E(D) \rightarrow \Gamma$ is called a $\Gamma$-flow if the usual conservation law (Kirchhoff's law) is satisfied, that is, for each vertex $v$, the sum of $\mathbf{f}(e)$ over all the half edges $(v, e)$ in $E^{+}(v)$ equals that in $E^{-}(v)$, i.e.,

$$
\sum_{(v, e) \in E^{+}(v)} \mathbf{f}(e)=\sum_{(v, e) \in E^{-}(v)} \mathbf{f}(e) .
$$

A flow $\mathbf{f}$ is called nowhere-zero if $\mathbf{f}(e) \neq 0$ for each $e \in E(D)$. It is straightforward to see that the number of nowhere-zero $\Gamma$-flows is independent of the orientation of $G$. A signed graph is said to be $\Gamma$-flow admissible if it admits at least one nowhere-zero $\Gamma$-flow. It is clear that the property of ' $\Gamma$-flow admissible' is invariant under switching inversion.

\section{Fundamental circuits in a signed graph}

In this section we generalize the notion of fundamental circuits in graphs to signed graphs, which will play an important role in revealing the structural properties of $\Gamma$-flows in signed graphs.

For a signed graph $G$ and a set $F$ of edges, we denote by $G+F$ and $G-F$ the subgraphs obtained from $G$ by adding and deleting the edges in $F$, respectively. Let $E_{N}=\left\{e_{0}, e_{1}, e_{2}, \cdots, e_{m_{N}-1}\right\}$ (the set of all negative edges of $G$ ), where $m_{N}=\left|E_{N}\right|$. In this section we always assume that $G$ is unbalanced and, with no loss of generality, contains as few negative edges as possible in its switching equivalent class. Thus, $E_{N} \neq \emptyset$ and $G-E_{N}$ is connected [20].

Let $T$ be a spanning tree of $G-E_{N}$. Choose an arbitrary edge $e_{0}$ from $E_{N}$ and call $T_{0}=T+e_{0}$ a signed rooted tree of $G$ with root edge $e_{0}$ (note that a signed rooted tree we defined here is not a tree in the usual sense for graphs because it has a unique unbalanced circuit). Let $\bar{T}_{0}=E(G) \backslash E\left(T_{0}\right)$. For any $e \in \bar{T}_{0}$, it is clear that $T_{0}+e$ contains a unique signed circuit. We call this circuit a fundamental circuit and denote it by $C_{e}$. We can see that, if $e \in \bar{T}_{0} \backslash E_{N}$ then $C_{e}$ is a graph circuit (a circuit without negative edge) and if $e \in E_{N} \backslash\left\{e_{0}\right\}$ then $C_{e}$ is a barbell or a balanced circuit with two negative edges $e_{0}$ and $e$.

For a barbell consisting of two unbalanced circuits $u_{1} e_{1} u_{2} \cdots u_{p} e_{p} u_{1}, v_{1} e_{1}^{\prime} v_{2} \cdots v_{q} e_{q}^{\prime} v_{1}$ and a barbell path $u_{1} e_{1}^{\prime \prime} w_{2} \cdots w_{r} e_{r}^{\prime \prime} v_{1}$, we also write it as a closed walk [10]:

$$
u_{1} e_{1} u_{2} \cdots u_{p} e_{p} u_{1} e_{1}^{\prime \prime} w_{2} \cdots w_{r} e_{r}^{\prime \prime} v_{1} e_{1}^{\prime} v_{2} \cdots v_{q} e_{q}^{\prime} v_{1} e_{r}^{\prime \prime} w_{r} \cdots w_{2} e_{1}^{\prime \prime} u_{1}
$$

Given a fixed orientation $D=\tau(G)$, a fundamental directed circuit $\vec{C}_{e}$ of $G$ is the orientation $\tau^{\prime}$ of a fundamental circuit $C_{e}=v_{1} e_{1} v_{2} e_{2} \cdots v_{k} e_{k} v_{1}$ (where $e=e_{1}=v_{1} v_{2}$ ) such that the direction of $e$ is the same as that which it has in $D$ and the directions of all other edges on $\vec{C}_{e}$ coincide consistently with $e$ along with $C_{e}$, i.e., $\tau^{\prime}\left(e_{1}\right)=\tau\left(e_{1}\right)$ and $\tau^{\prime}\left(v_{i}, e_{i-1}\right) \tau^{\prime}\left(v_{i}, e_{i}\right)=-1, \tau^{\prime}\left(v_{i}, e_{i}\right) \tau^{\prime}\left(v_{i+1}, e_{i}\right)=-\sigma\left(e_{i}\right)$ for every $i \in\{1,2, \cdots, k\}(\bmod k)$. Under this orientation, it can be seen that if $C_{e}$ is an ordinary circuit then $\vec{C}_{e}$ is a usual 
directed circuit with direction coincident with $D$ on $e$, and if $C_{e}$ is a balanced circuit or a barbell (with two negative edges $e_{0}$ and $e$ ), then the direction of the two negative edges are always opposite, that is, $e_{0}$ is extroverted if and only if $e$ is introverted, see Figure 1.

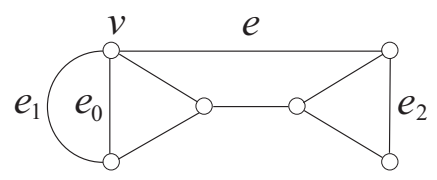

$G$

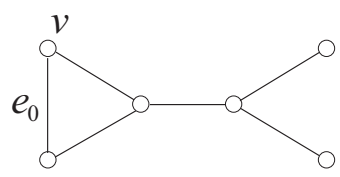

$T_{0}$

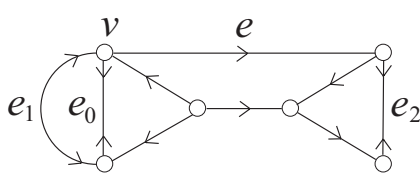

$D$

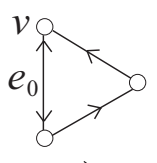

$\vec{C}_{0}$

Figure 1. The edges $e_{0}, e_{1}, e_{2}$ are negative and $e$ is positive.

For a fundamental circuit $C_{e}$, let $C_{e}^{D}$ be the orientation $D$ restricted to $C_{e}$. We associate with $C_{e}$ a function $\mathbf{f}_{e}$ on $E(D)$ defined by

$$
\mathbf{f}_{e}(a)=\left\{\begin{aligned}
1, & \text { if } a \in \vec{C}_{e} ; \\
-1, & \text { if } a \in C_{e}^{D} \backslash \vec{C}_{e} ; \\
2, & \text { if } a \in \vec{C}_{e} \text { and } a \text { is on the barbell path of } C_{e} ; \\
-2, & \text { if } a \in C_{e}^{D} \backslash \vec{C}_{e} \text { and } a \text { is on the barbell path of } C_{e} ; \\
0, & \text { otherwise }
\end{aligned}\right.
$$

for any $a \in E(D)$, where ' $a$ is on the barbell path of $C_{e}$ ' means that $C_{e}$ is a barbell and $a$ is an edge belonging to the barbell path of $C_{e}$.

From the above definition, it can be seen that $\mathbf{f}_{e}(e)=1$ for any $e \in \bar{T}_{0}$.

Let $C_{0}$ be the unique (un-balanced) circuit in $T_{0}$ (i.e., formed by $e_{0}$ and $T$ ). Choose an arbitrary vertex $v$ on $C_{0}$ and let $\vec{C}_{0}$ be the orientation of $C_{0}$ such that the direction of $e_{0}$ is extroverted and all other edges on $C_{0}$ are oriented so that $d^{-}(v)=2, d^{+}(v)=0$ and $d^{-}(u)=d^{+}(u)=1$ for any vertex $u$ on $C_{0}$ other than $v$, where $d^{-}(v)$ and $d^{+}(v)$ are the in-degree and out-degree of $v$ on $\vec{C}_{0}$, respectively, see Figure 1 . We call $\vec{C}_{0}$ the fundamental root circuit and associate it with a function $\mathbf{g}$ on $E(D)$ defined by

$$
\mathbf{g}(e)=\left\{\begin{aligned}
1, & \text { if } e \in \vec{C}_{0} ; \\
-1, & \text { if } e \in C_{0}^{D} \backslash \vec{C}_{0} \\
0, & \text { otherwise }
\end{aligned}\right.
$$

for any $e \in E(D)$.

For convenience, in the following we regard each $\Gamma$-flow, each function $\mathbf{f}_{e}\left(e \in \bar{T}_{0}\right)$ and the function $\mathbf{g}$ as $m$-dimensional vectors indexed by $e \in E(G)$. Let $\mathcal{S}_{G}$ denote the class of all $\Gamma$-flows (not necessarily nowhere-zero) in $G$. 
For a finite additive Abelian group $\Gamma$, let $\Gamma_{2}$ be the set of the elements of order 2 in $\Gamma$ (including the zero element). Recalling that $\epsilon(\Gamma)$ is the largest integer $d$ for which $\Gamma$ has a subgroup isomorphic to $\mathbb{Z}_{2}^{d}$, we have $\left|\Gamma_{2}\right|=2^{\epsilon(\Gamma)}$.

Theorem 1. Let $\Gamma$ be an additive Abelian group and let $G$ be a connected unbalanced signed graph. Let $T$ be a spanning tree of $G$ consisting of positive edges and let $e_{0} \in E_{N}$. Then

$$
\mathcal{S}_{G}=\left\{\gamma \mathbf{g}+\sum_{e \in \bar{T}_{0}} \gamma_{e} \mathbf{f}_{e}: \gamma \in \Gamma_{2}, \gamma_{e} \in \Gamma\right\}
$$

Proof. It is clear that

$$
\gamma \mathbf{g}+\sum_{e \in \bar{T}_{0}} \gamma_{e} \mathbf{f}_{e}
$$

is a $\Gamma$-flow for any $\gamma \in \Gamma_{2}$ and $\gamma_{e} \in \Gamma$. Let $\mathbf{f}$ be an arbitrary $\Gamma$-flow in $G$. We need only prove that $\mathbf{f}$ can be written as the combination (2).

Since a $\Gamma$-flow is independent of the orientation $D$, to simplify our discussion we make the following assumption:

Assumption 1. In orientation $D$, the direction of the root edge $e_{0}$ is extroverted while the directions of all other negative edges are introverted.

For each negative edge $e_{i}=u_{i} v_{i} \in E_{N}$, insert a new vertex $w_{i}$ into the middle of $e_{i}$ so that the two half edges of $e_{i}$ in $D$ become two ordinary directed edges $w_{i} u_{i}$ (with direction from $w_{i}$ to $u_{i}$ ) and $w_{i} v_{i}$ if $i=0$, or $u_{i} w_{i}$ and $v_{i} w_{i}$ if $i \in\left\{1,2, \cdots, m_{N}-1\right\}$. Further, add a new vertex $w$ to $D$ and add the directed edges $e_{0}^{\prime}=w w_{0}$ and $e_{i}^{\prime}=w_{i} w$ for every $i \in\left\{1,2, \cdots, m_{N}-1\right\}$. The resulting graph, denoted by $D^{w}$, is a directed graph without negative edges, that is, $D^{w}$ is an ordinary directed graph.

Let $\mathbf{f}^{w}$ be the extension of the function $\mathbf{f}$ from the edges of $D$ to the edges of $D^{w}$ defined by $\mathbf{f}^{w}\left(w_{0} u_{0}\right)=\mathbf{f}^{w}\left(w_{0} v_{0}\right)=\mathbf{f}\left(e_{0}\right), \mathbf{f}^{w}\left(e_{0}^{\prime}\right)=2 \mathbf{f}\left(e_{0}\right)$ and $\mathbf{f}^{w}\left(u_{i} w_{i}\right)=\mathbf{f}^{w}\left(v_{i} w_{i}\right)=\mathbf{f}\left(e_{i}\right)$, $\mathbf{f}^{w}\left(e_{i}^{\prime}\right)=2 \mathbf{f}\left(e_{i}\right)$ for $i \in\left\{1,2, \cdots, m_{N}-1\right\}$. It is clear that, except possibly for $w$, the conservation law is satisfied at all the vertices in $D^{w}$ and therefore must be also satisfied at $w$. Thus, by the conservation law at $w$, we have

$$
\mathbf{f}^{w}\left(e_{0}^{\prime}\right)=\sum_{i=1}^{m_{N}-1} \mathbf{f}^{w}\left(e_{i}^{\prime}\right)
$$

or equivalently,

$$
2 \mathbf{f}\left(e_{0}\right)=\sum_{i=1}^{m_{N}-1} 2 \mathbf{f}\left(e_{i}\right)=2 \sum_{e_{i} \in E_{N}^{*}} \mathbf{f}\left(e_{i}\right),
$$

where $E_{N}^{*}=E_{N} \backslash\left\{e_{0}\right\}=\left\{e_{1}, e_{2}, \cdots, e_{m_{N}-1}\right\}$.

Further, we note that, for any $\gamma \in \Gamma$, the solution of the equation $2 x=2 \gamma$ (in $x$ ) over $\Gamma$ has the form $x=\gamma+\gamma_{2}$, where $\gamma_{2}$ is an element of order 2 (possibly the zero element), 
i.e., $\gamma_{2} \in \Gamma_{2}$. Thus, (3) is equivalent to

$$
\mathbf{f}\left(e_{0}\right)=\gamma_{2}+\sum_{e_{i} \in E_{N}^{*}} \mathbf{f}\left(e_{i}\right)
$$

where $\gamma_{2} \in \Gamma_{2}$.

On the other hand, for any $e \in E_{N}^{*}$, by Assumption 1 and the definitions of $\vec{C}_{e}$ and $\mathbf{f}_{e}$, we have

$$
\mathbf{f}_{e}\left(e_{0}\right)=\mathbf{f}_{e}\left(e_{i}\right)=1 \text {. }
$$

In (2), we set $\gamma=\gamma_{2}$ and for $e \in \bar{T}_{0}$, set $\gamma_{e}=\mathbf{f}(e)$. Let

$$
\mathbf{f}^{\prime}=\mathbf{f}-\left(\gamma_{2} \mathbf{g}+\sum_{e \in \bar{T}_{0}} \gamma_{e} \mathbf{f}_{e}\right)
$$

Then for any $e \in \bar{T}_{0}$, by the definition of the vector $\mathbf{g}$ we have $\gamma_{2} \mathbf{g}(e)=0$ since $e$ is not on $C_{0}$. This implies that $\mathbf{f}^{\prime}(e)=0$ for any $e \in \bar{T}_{0}$ because $\gamma_{e}=\mathbf{f}(e)$ and, as mentioned earlier, $\mathbf{f}_{e}(e)=1$. Further, by (4), (5) and (6) we have

$$
\begin{aligned}
\mathbf{f}^{\prime}\left(e_{0}\right) & =\mathbf{f}\left(e_{0}\right)-\left(\gamma_{2} \mathbf{g}\left(e_{0}\right)+\sum_{e \in \bar{T}_{0}} \gamma_{e} \mathbf{f}_{e}\left(e_{0}\right)\right) \\
& =\gamma_{2}+\sum_{e \in E_{N}^{*}} \mathbf{f}(e)-\left(\gamma_{2} \mathbf{g}\left(e_{0}\right)+\sum_{e \in \bar{T}_{0} \backslash E_{N}^{*}} \gamma_{e} \mathbf{f}_{e}\left(e_{0}\right)+\sum_{e \in E_{N}^{*}} \gamma_{e} \mathbf{f}_{e}\left(e_{0}\right)\right) \\
& =\sum_{e \in E_{N}^{*}} \mathbf{f}(e)-\sum_{e \in E_{N}^{*}} \gamma_{e} \mathbf{f}_{e}\left(e_{0}\right) \\
& =\sum_{e \in E_{N}^{*}} \mathbf{f}(e)\left(1-\mathbf{f}_{e}\left(e_{0}\right)\right) \\
& =0
\end{aligned}
$$

where the third equality holds because $\mathbf{g}\left(e_{0}\right)=1$ and $e_{0} \notin C_{e}$ for any $e \in \bar{T}_{0} \backslash E_{N}^{*}$ and, therefore $\mathbf{f}_{e}\left(e_{0}\right)=0$; and the last two equalities hold because of (5) and $\gamma_{e}=\mathbf{f}(e)$ for any $e \in E_{N}^{*}$.

The above discussion means that $\mathbf{f}^{\prime}$ evaluated at each edge outside of $T$ is zero. Thus, we must have $\mathbf{f}^{\prime}=\mathbf{0}$ (the vector of all zeros) because the values of $\mathbf{f}^{\prime}$ at the edges of $T$ are uniquely determined by those outside $T$. In conclusion, $\mathbf{f}$ is represented as the combination (2), which completes our proof.

\section{Classification of $\Gamma$-flows in a signed graph}

From Theorem 1, we know that all $\Gamma$-flows in a connected unbalanced signed graph can be 'generated' by fundamental root circuit $\vec{C}_{0}$ and the fundamental directed circuits $\vec{C}_{e}, e \in \bar{T}_{0}$. This leads to the following classification of $\Gamma$-flows in a signed graph, which are specified by the elements of order 2 in $\Gamma$.

Theorem 2. Let $\Gamma$ be an additive Abelian group of order $k$ and let $G$ be a connected unbalanced signed graph. Let $T$ be a spanning tree of $G$ consisting of positive edges and 
let $e_{0} \in E_{N}$.

1). The flows in $\mathcal{S}_{G}$ are pairwise distinct and, therefore

$$
\left|\mathcal{S}_{G}\right|=2^{\epsilon(\Gamma)} k^{m-n}
$$

2). $\mathcal{S}_{G}$ can be evenly partitioned into $\left|\Gamma_{2}\right|$ classes specified by the elements in $\Gamma_{2}$, i.e., $\mathcal{S}_{G}=\bigcup_{\gamma \in \Gamma_{2}} \mathcal{S}_{G}(\gamma)$ and $\left|\mathcal{S}_{G}(\gamma)\right|=k^{m-n}$ for any $\gamma \in \Gamma_{2}$, where

$$
\mathcal{S}_{G}(\gamma)=\left\{\gamma \mathbf{g}+\sum_{e \in \bar{T}_{0}} \gamma_{e} \mathbf{f}_{e}: \gamma_{e} \in \Gamma\right\}
$$

Proof. 1). We need only prove that

$$
\gamma \mathbf{g}+\sum_{e \in \bar{T}_{0}} \gamma_{e} \mathbf{f}_{e}=\gamma^{\prime} \mathbf{g}+\sum_{e \in \bar{T}_{0}} \gamma_{e}^{\prime} \mathbf{f}_{e}
$$

if and only if $\gamma=\gamma^{\prime}$ and $\gamma_{e}=\gamma_{e}^{\prime}$ for any $e \in \bar{T}_{0}$. For any $e \in \bar{T}_{0}$, by the definition of $\mathbf{g}$ and $\mathbf{f}_{e}$ we have $\mathbf{f}_{e}(e)=1, \mathbf{g}(e)=0$ and $\mathbf{f}_{e^{\prime}}(e)=0$ for any $e^{\prime} \in \bar{T}_{0}$ with $e^{\prime} \neq e$. Thus, (9) implies that $\gamma_{e} \mathbf{f}_{e}(e)=\gamma_{e}^{\prime} \mathbf{f}_{e}(e)$ and, therefore $\gamma_{e}=\gamma_{e}^{\prime}$ for any $e \in \bar{T}_{0}$. Consequently, again by (9), we have $\gamma \mathbf{g}=\gamma^{\prime} \mathbf{g}$ and, therefore $\gamma=\gamma^{\prime}$.

2 ). Since the flows in $\mathcal{S}_{G}$ are pairwise distinct, 2) follows directly.

For a component $\omega$ of a signed graph $G$, let

$$
\beta(\omega)=\left\{\begin{array}{cl}
m(\omega)-n(\omega)+1, & \text { if } \omega \text { is balanced } \\
m(\omega)-n(\omega), & \text { if } \omega \text { is unbalanced }
\end{array}\right.
$$

where $m(\omega)$ and $n(\omega)$ are the number of edges and vertices in $\omega$, respectively. In general, we let $\beta(G)=\sum \beta(\omega)$, where the sum is taken over all the components $\omega$ of $G$. Let $\kappa(G)$ be the number of unbalanced components and $F^{*}(G, \Gamma)$ be the number of $\Gamma$-flows (not necessarily nowhere-zero) in $G$.

Corollary 3. Let $G$ be a signed graph and let $\Gamma$ be an additive Abelian group of order $k$. Then

$$
F^{*}(G, \Gamma)=2^{\kappa(G) \epsilon(\Gamma)} k^{\beta(G)} .
$$

Proof. If $G$ is not connected then $F^{*}(G, \Gamma)=\prod F^{*}(\omega, \Gamma)$, where the product is taken over all the components $\omega$ of $G$. We need only consider the case when $G$ is connected.

If $G$ is unbalanced then (11) follows directly from (7). Now assume that $G$ is balanced. Recall that a balanced signed graph is switching-equivalent to an ordinary graph. In this case it is known [12] that the number of $\Gamma$-flows (not necessarily nowhere-zero) in an ordinary graph is $k^{m-n+1}$, i.e., $F^{*}(G, \Gamma)=k^{m-n+1}$, where $m$ and $n$ are the numbers of edges and vertices in $G$, respectively. This agrees with (11) because $\kappa(G)=0$ and $\beta(G)=m-n+1$ when $G$ is balanced. The proof is completed. 
Remark 1. When $k$ (the order of $\Gamma$ ) is odd, Beck and Zaslavsky posed a problem (Problem 4.2, [1]): Is there any significance to $F^{*}(G, \Gamma)$ evaluated at even natural numbers? By Theorem 2 and Corollary 3 we can now give an answer to this problem. For simplicity, let's consider the case when $G$ is connected and unbalanced. Since $k$ is odd, we have $\epsilon(\Gamma)=0$ and therefore, $F^{*}(G, \Gamma)=k^{m-n}$. Thus, $F^{*}(G, \Gamma)$ evaluated at an even number $h$ equals $h^{m-n}$, which is exactly the number of $\Gamma^{\prime}$-flows in $G$ divided by $2^{\epsilon\left(\Gamma^{\prime}\right)}$ for any group $\Gamma^{\prime}$ of order $h$. More specifically, by Theorem $2, F^{*}(G, \Gamma)$ evaluated at $h$ equals the number of those $\Gamma^{\prime}$-flows in $G$ which have the form

$$
\mathbf{f}=\gamma \mathbf{g}+\sum_{e \in \bar{T}_{0}} \gamma_{e} \mathbf{f}_{e}, \quad \gamma_{e} \in \Gamma^{\prime}
$$

where $\gamma$ is an arbitrary fixed element of order 2 in $\Gamma^{\prime}$ (in particular we may choose $\gamma=0$ ).

For any $e \in E(G)$, the number of $\Gamma$-flows in $G$ with value 0 at $e$ is clearly equal to $F^{*}(G-e, \Gamma)$. The polynomial $F_{d}(G, x)$ evaluated at $|\Gamma|$ counts the number of nowhere-zero $\Gamma$-flows. So by Corollary 3 and the principle of inclusion-exclusion, we get the following expression of $F_{d}(G, x)$ obtained earlier by Goodall et al.:

Corollary 4. [9] For any signed graph $G$ and non-negative integer d,

$$
F_{d}(G, x)=\sum_{F \subseteq E}(-1)^{|F|} 2^{\kappa(G-F) d} x^{\beta(G-F)} .
$$

We note that, if $G$ is an ordinary graph then $\kappa(G-F)=0$ for any $F \subseteq E(G)$. Therefore, Corollary 4 generalizes the corresponding result for ordinary graphs $[8,12]$.

Example. By Corollary 4, if $G$ is the graph with two vertices joined by a negative edge and a positive edge then $F_{d}(G, x)=2^{d}-1$; if $G$ is the graph consisting of two negative loops at a vertex then $F_{d}(G, x)=2^{d} x-2^{d+1}+1$; and if $G$ is the graph consisting of a negative loop and a positive loop at a vertex then $F_{d}(G, x)=\left(2^{d}-1\right)(x-1)$.

\section{Coefficients in $F_{0}(G, x)$}

In this section we will give a combinatorial interpretation of the coefficients in $F_{d}(G, x)$ for $d=0$. We begin with the following extension of Whitney's broken circuit theorem given by Dohmen and Trinks.

Lemma 5. [7] Let $P$ be a finite linearly ordered set, $\mathscr{B} \subseteq 2^{P} \backslash\{\emptyset\}$ and $\Gamma$ be an additive Abelian group. Let $f: 2^{P} \rightarrow \Gamma$ be a mapping such that, for any $B \in \mathscr{B}$ and $A \supseteq B$,

$$
f(A)=f(A \backslash\{\max B\}) .
$$

Then

$$
\sum_{A \in 2^{P}}(-1)^{|A|} f(A)=\sum_{A \in 2^{P} \backslash \mathscr{B}^{*}}(-1)^{|A|} f(A)
$$


where $\max B$ is the maximum element in $B$ and

$$
\mathscr{B}^{*}=\left\{A: A \in 2^{P}, A \supseteq B \backslash\{\max B\} \text { for some } B \in \mathscr{B}\right\} .
$$

We call $\mathscr{B}$ in Lemma 5 a broken system of $f$ and $B \backslash\{\max B\}$, or $B \backslash \max B$ for short, a broken set for any $B \in \mathscr{B}$.

To apply Lemma 5 we need to define a broken system and broken sets for signed graphs. We follow the idea of the notion of 'bonds' introduced in [5, 20]. For a signed graph $G$ and $X \subseteq V(G)$, denote by $\left[X, X^{C}\right]$ the set of edges between $X$ and its complement $X^{C}$, by $G[X]$ the subgraph of $G$ induced by $X$, and by $E(X)$ the set of the edges in $G[X]$. A non-empty edge subset $B \subseteq E(G)$ is called a cut [5] or improving set [20] of $G$ if it has the form $B=\left[X, X^{C}\right] \cup E_{X}$, where $X \subseteq V(G)$ is non-empty and $E_{X} \subseteq E(X)$ is minimal to have $G[X]-E_{X}$ balanced. A cut is called a bond of $G$ if it is minimal. We note that, in the case when $G$ is balanced, we have $E_{X}=\emptyset$ by the minimality of $E_{X}$ and, therefore a bond is exactly a usual bond as in an ordinary graph. In this sense, the notion 'bond' for signed graph is a very nice extension of that for ordinary graphs [12].

By the definition of a broken set, it is not difficult to see that if $B$ is a bond then, for any $e \in B$,

$$
\beta(G-B)=\beta(G-(B \backslash\{e\})) .
$$

On the other hand, by Corollary 4, we have

$$
F_{0}(G, x)=\sum_{F \subseteq E}(-1)^{|F|} x^{\beta(G-F)} .
$$

Thus, an edge subset of $G$ is a broken set for $F_{0}(G, x)$ if it has the form $B \backslash \max B$ for some $B \subseteq E(G)$ such that, for any $A \supseteq B$,

$$
\beta(G-A)=\beta(G-(A \backslash \max B)) .
$$

On the other hand, by (14), for any bond $B$ we have

$$
\beta(G-B)=\beta(G-(B \backslash \max B)) .
$$

Moreover, it is not difficult to see that, for any $A \supseteq B,(15)$ is satisfied by $A$ and $B$. Thus, $B \backslash \max B$ is a broken set for $F_{0}(G, x)$ for any bond $B$ and is called a broken bond of $G$. Then by Lemma 5 we immediately have the following result.

Theorem 6. For any signed graph $G$ with a linear order $\prec$ on $E(G)$,

$$
F_{0}(G, x)=\sum_{F \in 2^{E(G)} \backslash \mathscr{B}^{*}}(-1)^{|F|} x^{\beta(G-F)},
$$

where $\mathscr{B}$ is the class of bonds of $G$ and

$$
\mathscr{B}^{*}=\left\{F: F \in 2^{E(G)}, F \supseteq B \backslash \max B \text { for some } B \in \mathscr{B}\right\} .
$$


Remark 2. If $G$ is balanced, then each broken bond is exactly a usual broken bond of an ordinary graph. In this case, (16) is still valid. Thus, Theorem 6 is a generalization of that for ordinary graph [12]. Further, in a very special case when an unbalanced signed graph $G$ contains an edge whose removal leaves a balanced graph, the empty set is a broken bond and therefore any set of edges (including the empty set) contains a broken bond. This case means that $\mathscr{B}^{*}=2^{E(G)}$ and thus, $F_{0}(G, x)=0$, which coincides with the obvious fact that such a $G$ is not $\Gamma$-flow admissible when $|\Gamma|$ is odd.

Proposition 7. For any signed graph $G$ and $F \subseteq E(G)$, if $F$ contains no broken bond then each component of $G-F$ is unbalanced, unless $G$ is balanced.

Proof. To the contrary suppose that one component $\omega$ of $G-F$ is balanced. Let $B=$ $[V(\omega), \overline{V(\omega)}] \cup E_{F}$, where $E_{F}$ is the set of edges in $F$ whose two end vertices are both in $\omega$. Then $B$ is a bond since $\omega$ is balanced and thus $B \backslash \max B$ is a broken bond. Notice that $B \backslash \max B \subset B \subseteq F$, which contradicts that $F$ contains no broken bond.

Let $\eta(G)$ be the number of those edges $e$ such that there is an edge $e^{\prime}$ with $e \prec e^{\prime}$ satisfying one of the following three conditions:

$1)$. one of $e$ and $e^{\prime}$ is a cut edge and $G-\left\{e, e^{\prime}\right\}$ has a balanced component;

2). $\left\{e, e^{\prime}\right\}$ is an edge cut and $G-\left\{e, e^{\prime}\right\}$ has a balanced component;

3). $\left\{e, e^{\prime}\right\}$ is contained in a component $\omega$ of $G$ and $\omega-\left\{e, e^{\prime}\right\}$ is balanced.

Corollary 8. Let $G$ be an unbalanced, $\Gamma$-flow admissible $(|\Gamma|$ is odd) signed graph with $n$ vertices and $m$ edges. Then for any linear order $\prec$ on $E(G)$,

$$
F_{0}(G, x)=a_{0} x^{m-n}-a_{1} x^{m-n-1}+a_{2} x^{m-n-2}-\cdots+(-1)^{m-n} a_{m-n},
$$

where, for each $i \in\{0,1, \cdots, m-n\}, a_{i}$ is the number of edge subsets of $G$ having $i$ edges and containing no broken bond as a subset. In particular,

1). $a_{i}>0$ for every $i=0,1,2, \cdots, m-n$;

2). $a_{0}=1$;

3). $a_{1}=m-\eta(G)$;

Proof. Let $F \subseteq E(G)$ be an edge subset that contains no broken bond. Since $G$ is unbalanced, by Proposition 7 every component $\omega$ of $G-F$ is unbalanced. Thus, $\beta(\omega)=$ $m(\omega)-n(\omega)$ due to (10). Therefore,

$$
\beta(G-F)=\sum_{\omega} \beta(\omega)=m(G-F)-n(G-F)=m-n-|F|,
$$

where the sum is taken over all the components of $G-F$. This equation means that the value of $\beta(G-F)$ is determined uniquely by the number of edges in $F$, as long as $F$ contains no broken bond. So by Theorem 6 , the coefficient of $(-1)^{i} x^{m-n-i}$ in $F_{0}(G, x)$ counts exactly those edge subsets $F$ which have $i$ edges and contain no broken bond. Thus, (17) follows directly.

$1)$. We first show that there is an edge set $F$ with $n$ edges that contains no broken bond. By the definition of a broken bond, an edge set $F$ contains no broken bond if and only if 
$E(G) \backslash F$ contains at least one edge from each broken bond of $G$. Let $F^{*}$ be maximum such that $E(G) \backslash F^{*}$ contains at least one edge from each broken bond of $G$ (such $F^{*}$ clearly exists because $E(G) \backslash \emptyset$ does). Let $\omega$ be a component of $G-F^{*}$. Then by Proposition 7 , $\omega$ contains at least one unbalanced circuit, say $C_{u}$. We claim that $\omega$ does not contain any other circuit.

Suppose to the contrary that $C$ is a circuit in $\omega$ with $C \neq C_{u}$. Since $C$ is a circuit, the property that $G-F^{*}$ contains at least one edge from each broken bond is still satisfied by $G-F^{*}-\max C$ because any bond containing $\max C$ must contain another edge $e$ on $C$ with, of course, $e \prec \max C$. This contradicts our assumption that $F^{*}$ is maximum. Our claim follows.

In a word, each component $\omega$ of $G-F^{*}$ contains exactly one unbalanced circuit and no any other circuit. This means that $m(\omega)=n(\omega)$ and, therefore $m\left(G-F^{*}\right)=n$, i.e., $\left|F^{*}\right|=m-n$. Thus, $a_{m-n}>0$. Further, if an edge subset $F$ contains no broken bond then any subset of $F$ contains neither broken bond, which implies $a_{i}>0$ for any $i$ with $0 \leqslant i \leqslant m-n$.

2 ). Since $G$ is flow-admissible, as pointed out in Remark 2, $G$ contains no edge whose removal leaves a balanced graph. This means that the empty set is not a broken bond. Thus, $a_{0}$ equals the number of the edge subsets of $G$ having 0 edges, that is, the unique empty set.

3 ). Now we consider the coefficient $a_{1}$. From the above discussion we see that $a_{1}$ equals the number of the edges that are not broken bond. On the other hand, an edge $e$ is a broken bond if there is $e^{\prime}$ such that $B=\left\{e, e^{\prime}\right\}$ is a bond and $e^{\prime}=\max B$. By the definition of a bond, $B=\left\{e, e^{\prime}\right\}$ must satisfy one of the above three conditions and, vice versa.

Remark 3. Corollary 8 remains a natural question: How about the case when $|\Gamma|$ is even? Indeed, by Lemma 5 and Corollary 4 , if an edge subset is a broken set for $F_{d}(G, x)$ then it must have the form of $B \backslash \max B$ satisfying both (15) and

$$
\kappa(G-A) d=\kappa(G-(A \backslash \max B)) d
$$

for any $A \supseteq B$. When $d=0,(18)$ always holds and, hence the family of broken sets can be chosen to be the one consisting of all broken bonds since (15) is satisfied by every broken bond. For $d>0$, it seems not easy to find such a $B$ that satisfy both (15) and (18), unless the signed graph $G$ admits some particular properties as shown in the following, for an example:

For a bond $B=\left[X, X^{C}\right] \cup E_{X}$, it is not difficult to see that if $\max B \in\left[X, X^{C}\right]$ then $\kappa(G-A) d=\kappa(G-(A \backslash \max B)) d$ for any $A \supseteq B$. In this case, $B \backslash \max B$ is also a broken set for $F_{d}(G, x)$ with arbitrary nonnegative integer $d$. This implies that if $G$ has an edge-ordering such that $\max B \in\left[X, X^{C}\right]$ for every bond $B$ then the broken bond expansion (16) for $F_{0}(G, x)$ can be generalized to

$$
F_{d}(G, x)=\sum_{F \in 2^{E(G)} \backslash \mathscr{B}^{*}}(-1)^{|F|} 2^{\kappa(G-F) d} x^{\beta(G-F)}
$$


for any $d \geqslant 0$. Even so, it does not mean that (19) can yield an interpretation for the coefficients of $F_{d}(G, x)$, unless $\kappa(G-F)$ is determined uniquely by the number of edges in $F$, for an example (in the following section we will give such an example).

\section{Applications}

The broken bond expansions in Corollary 8 and (19) allow us to calculate $F_{0}(G, x)$ and $F_{d}(G, x)$ for some particular signed graphs. For our first application, we consider a class of signed graphs which, oppositely to ordinary graphs, do not contain any balanced circuit, and which are $\Gamma$-flow admissible.

For a tree $T$, let $G_{T}$ be the signed graph obtained from $T$ by replacing each of its end vertices (the vertices of degree 1 ) with an unbalanced circuit. It is clear that $G_{T}$ contains no balanced circuit.

Let $v_{1}, v_{2}, \cdots, v_{p}$ be the vertices in $T$ that have degree at least 3 and let $d_{1}, d_{2}, \cdots, d_{p}$ be their degrees, respectively. Choosing an arbitrary leaf vertex $r$ of $T$ as the root, we get a rooted tree (here the 'rooted tree' is not the same thing as the 'signed rooted tree' defined earlier). For a vertex $v_{i}$ (with degree at least 3) and an edge $e$ incident with $v_{i}$, we call $e$ the father of the family $v_{i}$ if $e$ is nearer to the root than other edges incident with $v_{i}$ and call every edge other than the father a child of the family $v_{i}$. In particular, we call the set of all the children of $v_{i}$ the children class of $v_{i}$ and denote it by $C\left(v_{i}\right)$.

Let $\prec$ be an ordering on $E\left(G_{T}\right)$ such that no child is greater than its father and no edge on an unbalanced circuit is greater than one on $T$. Under this ordering, we can see that $\max B$ must be an edge on $T$ for any bond $B=\left[X, X^{C}\right] \cup E_{X}$ in $G_{T}$, meaning that $\max B \in\left[X, X^{C}\right]$. Thus, the expansion (19) holds for $G_{T}$. Further, in the following we give an analytic expression of $F_{0}\left(G_{T}, x\right)$ for any tree $T$ and expression of $F_{d}\left(G_{T}, x\right)$ when $T$ is a star.

Let $F$ be an edge set of $G_{T}$ that contains no broken bond. By Corollary 8, $F$ contributes $(-1)^{|F|} x^{m-n-|F|}$ to $F_{0}\left(G_{T}, x\right)$, where $m=\left|E\left(G_{T}\right)\right|, n=\left|V\left(G_{T}\right)\right|$. On the other hand, by our definition of $\prec, F$ contains no broken bond if and only if $F$ contains neither an edge from an unbalanced circuit nor a children class of a family. For any vertex $v_{i}$, let $F_{i}=F \cap C\left(v_{i}\right)$. In particular, let $F_{r}=F \cap\left\{e_{r}\right\}$, where $e_{r}$ is the unique edge incident with the root $r$. Thus, the contribution of $F$ to $F_{0}\left(G_{T}, x\right)$ can be specified as

$$
x^{m-n}(-1)^{\left|F_{r}\right|} x^{-\left|F_{r}\right|} \prod_{i=1}^{p}(-1)^{\left|F_{i}\right|} x^{-\left|F_{i}\right|} \text {. }
$$

On the other hand, we notice that $m-n=\left(d_{1}-2\right)+\left(d_{2}-2\right)+\cdots+\left(d_{p}-2\right)+1$. Rewrite (20) as

$$
(-1)^{\left|F_{r}\right|} x^{1-\left|F_{r}\right|} \prod_{i=1}^{p}(-1)^{\left|F_{i}\right|} x^{d_{i}-2-\left|F_{i}\right|} .
$$

In this product, the factors $(-1)^{\left|F_{r}\right|} x^{1-\left|F_{r}\right|}$ and $(-1)^{\left|F_{i}\right|} x^{d_{i}-2-\left|F_{i}\right|}$ can be regarded as the contributions of $F$ restricted to $\left\{e_{r}\right\}$ and $C\left(v_{i}\right)$, respectively. Since $F \cap\left\{e_{r}\right\}=\emptyset$ or 
$F \cap\left\{e_{r}\right\}=\left\{e_{r}\right\}$, all the possible contributions of $F$ restricted to $\left\{e_{r}\right\}$ can be represented as $(-1)^{|\emptyset|} x^{1-|\emptyset|}+(-1)^{\left|\left\{e_{r}\right\}\right|} x^{1-\left|\left\{e_{r}\right\}\right|}=x-1$.

In general, for each $i$, since $v_{i}$ has exactly $d_{i}-1$ children, all the possible contributions of $F$ restricted to $C\left(v_{i}\right)$ equals

$$
x^{d_{i}-2}-\left(\begin{array}{c}
d_{i}-1 \\
1
\end{array}\right) x^{d_{i}-3}+\cdots+(-1)^{d_{i}-2}\left(\begin{array}{c}
d_{i}-1 \\
d_{i}-2
\end{array}\right) .
$$

Thus, the total contribution of all $F$ that contains no broken bond equals

$$
F_{0}\left(G_{T}, x\right)=(x-1) \prod_{i=1}^{p}\left(x^{d_{i}-2}-\left(\begin{array}{c}
d_{i}-1 \\
1
\end{array}\right) x^{d_{i}-3}+\cdots+(-1)^{d_{i}-2}\left(\begin{array}{c}
d_{i}-1 \\
d_{i}-2
\end{array}\right)\right) .
$$

When $T$ is a star, every edge on an unbalanced circuit of $G_{T}$ is a broken bond. Therefore, the number of unbalanced components in $G_{T}-F$ is determined uniquely by $|F|$, i.e., $\kappa\left(G_{T}-F\right)=|F|+1$. So by (19), the coefficient of $(-1)^{i} x^{m-n-i}$ in $F_{d}\left(G_{T}, x\right)$ equals $2^{(i+1) d} a_{i}$, where $i \in\{0,1,2, \cdots, m-n\}$ and $a_{i}$ is defined as in Corollary 8. Further, since $T$ is a star, we have $p=1$ in $(21)$ and, hence $a_{0}=1, a_{d_{1}-1}=\left(\begin{array}{c}d_{1}-1 \\ d_{1}-2\end{array}\right)$ and $a_{i}=\left(\begin{array}{c}d_{1}-1 \\ i-1\end{array}\right)+\left(\begin{array}{c}d_{1}-1 \\ i\end{array}\right)$ for $i \in\left\{1,2, \cdots, d_{1}-2\right\}$.

Our second application is to show that the broken bonds in a signed graph have the topological structure of a homogeneous simplicial complex. A finite collection $\mathscr{S}$ of finite sets is called a simplicial complex if $S \in \mathscr{S}$ implies $T \in \mathscr{S}$ for any $T \subseteq S$. A simplicial complex is homogeneous [19] or pure [3] if all the maximal simplices have the same dimension (cardinality). A classic example of a homogeneous simplicial complex related to a graph is the broken-circuit complex [3, 4]. It has been shown [19] that the class $\mathfrak{B}(G)$ consisting of all the edge subsets of an ordinary graph $G$ that contain no broken circuit is a homogeneous simplicial complex of top dimension $|V(G)|-1$ and, moreover, the coefficients of the chromatic polynomial of $G$ are the simplex counts in each dimension of $\mathfrak{B}(G)$.

Let $\mathfrak{F}(G)$ be the class consisting of all the edge subsets of a signed graph $G$ that contain no broken bond.

Corollary 9. Let $G$ be an unbalanced signed graph with $n$ vertices, $m$ edges and with a linear order $\prec$ on $E(G)$. Then

1). $\mathfrak{F}(G)$ is a homogeneous simplicial complex, i.e., every simplex is a subset of some simplex of top dimension $m-n$;

2). An edge set $F$ is a simplex of top dimension $m-n$ of $\mathfrak{F}(G)$ if and only if $E(G) \backslash F$ contains at least one edge from each broken bond of $G$ and each component $G-F$ contains exactly one unbalanced circuit;

3). For each $i \in\{0,1,2, \cdots, m-n\}$, the coefficient $a_{i}$ in $F_{0}(G, x)$ is the number of the $i$-dimensional simplexes in $\mathfrak{F}(G)$.

Proof. 1). It is obvious that $\mathfrak{F}(G)$ is a simplicial complex. We prove that $\mathfrak{F}(G)$ is homogeneous. 
Let $F$ be a set of edges that contains no broken bond. If $|F|=m-n$ then we are done. We now assume that $|F|<m-n$, i.e., $|E(G-F)|>n$. In this case, it can be seen that there is a component $\omega$ in $G-F$ which contains at least two circuits $C$ and $C^{\prime}$. By Proposition 7 , one of these two circuits, say $C$, is unbalanced. So by the same argument as that in Corollary 8, we can find an edge $e$ in $C^{\prime}$ such that $G-F-e$ still contains an edge from each broken bond. Replacing $F$ by $F \cup\{e\}$, the assertion follows by repeating this procedure, until $|F|=m-n$.

2) and 3) follows directly by Corollary 8.

\section{Acknowledgements}

The authors would like to thank the anonymous reviewer for her/his careful reading of our original manuscript and constructive suggestions which is very helpful for revising and improving the manuscript.

\section{References}

[1] M. Beck and T. Zaslavsky. The number of nowhere-zero flows on graphs and signed graphs. J. Combin. Theory, Ser. B, 96:901-918, 2006.

[2] A. Bouchet. Nowhere-zero integral flows on a bidirected graph. J. Combin. Theory, Ser. B, 34(3):279-292, 1983.

[3] T. Brylawski. The broken-circuit complex. Trans. Amer. Math. Soc., 234(2):417-433, 1977.

[4] T. Brylawski and J. Oxley. The broken-circuit complex: Its structure and factorizations. European J. Combin., 2(2):107-121, 1981.

[5] B. F Chen and J. Wang. The flow and tension spaces and lattices of signed graphs. European J. Combin., 30:263-279, 2009.

[6] M. DeVos, E. Rollová, and R. Sámal. A note on counting flows in signed graphs. Electron J. Combin., 26(2):\#P2.38, 2019.

[7] K. Dohmen and M. Trinks. An abstraction of Whitney's broken circuit theorem. Electron J. Combin., 21(4):\#P4.32, 2014.

[8] F. M. Dong and K. M. Koh. Bounds for the coefficients of flow polynomials. J. Combin. Theory, Ser B, 97:413-420, 2007.

[9] A. Goodall, B. Litjens, G. Regts, and L. Vena. A Tutte polynomial for maps II: the non-orientable case. arXiv:1804.01496v1, 2018.

[10] A. Goodall, B. Litjens, G. Regts, and L. Vena. Tutte's dichromate for signed graphs. arXiv:1903.07548, 2019.

[11] F. Harary. On the notion of balance of a signed graph, Michigan Math. J, 2(2):143146, 1953-1954.

[12] X.A. Jin. On the coefficients of the flow polynomial. J. Xinjiang Uni, 23:53-57, 2006. 
[13] T. Kaiser, R. Lukot'ka, and E. Rollová. Nowhere-zero flows in signed graphs: A survey. arXiv:1608.06944, 2016.

[14] Martin Kochol. Polynomials associated with nowhere-zero flows. J. Combin. Theory, Ser. B, 84:260-269, 2002.

[15] A. Raspaud and X. D. Zhu. Circular flow on signed graphs. J. Combin. Theory, Ser. B, 101:464-479, 2011.

Handbook of Combinatorics, vol. 1, North-Holland (Elsevier), Amsterdam, 1995.

[16] W. T. Tutte. On the imbedding of linear graphs in surfaces. Proc. London Math. Soc., 51(2):474-483, 1949.

[17] W. T. Tutte. A contribution to the theory of chromatic polynomials. Canad. J. Math., 6:80-91, 1954.

[18] H. Whitney. A logical expansion in mathematics. Bull. Amer. Math. Soc., 38(8):572$579,1932$.

[19] H. Wilf. Which polynomials are chromatic. Proc. Colloq. Combin Theory, Rome. 1973.

[20] T. Zaslavsky. Signed graphs. Discrete Appl. Math., 4:47-74, 1982.

[21] T. Zaslavsky. Orientation of signed graphs. European J. Combin., 12:361-375, 1991.

[22] T. Zaslavsky. Signed graph coloring. Discrete Math., 35:215-228, 1982.

[23] Y. Lu, J. Cheng, R. Luo, and C. Q. Zhang. Shortest circuit covers of signed graphs. J. Combin. Theory, Ser. B, 134:164-178, 2018. 